\title{
Transplanted cord blood-derived endothelial precursor cells augment postnatal neovascularization
}

\author{
Toyoaki Murohara,,$^{1,2}$ Hisao Ikeda, ${ }^{2}$ Junli Duan, ${ }^{1,2}$ Satoshi Shintani, ${ }^{1,2}$ Ken-ichiro Sasaki, ${ }^{1,2}$ \\ Hiroyuki Eguchi, ${ }^{1,2}$ Ichiro Onitsuka, ${ }^{2}$ Kazuo Matsui, ${ }^{3}$ and Tsutomu Imaizumi ${ }^{2}$
}

${ }^{1}$ The Cardiovascular Research Institute, and
${ }^{2}$ Department of Internal Medicine III, Kurume University School of Medicine, Kurume, Japan
${ }^{3}$ Department of Obstetrics and Gynecology, Fukuda Hospital, Kumamoto, Japan

Address correspondence to: Toyoaki Murohara, The Cardiovascular Research Institute, Kurume University, 67 Asahi-machi, Kurume 830-0011, Japan. Phone: 81-942-35-3311 ext. 3801; Fax: 81-942-31-7707; E-mail: toyom@med.kurume-u.ac.jp.

Received for publication August 30, 1999, and accepted in revised form April 10, 2000.

\begin{abstract}
Endothelial precursor cells (EPCs) have been identified in adult peripheral blood. We examined whether EPCs could be isolated from umbilical cord blood, a rich source for hematopoietic progenitors, and whether in vivo transplantation of EPCs could modulate postnatal neovascularization. Numerous cell clusters, spindle-shaped and attaching (AT) cells, and cord-like structures developed from culture of cord blood mononuclear cells (MNCs). Fluorescence-trace experiments revealed that cell clusters, AT cells, and cord-like structures predominantly were derived from CD34-positive MNCs (MNC $\left.{ }^{\mathrm{CD} 34+}\right)$. AT cells and cell clusters could be generated more efficiently from cord blood MNCs than from adult peripheral blood MNCs. AT cells incorporated acetylated-LDL, released nitric oxide, and expressed KDR, VE-cadherin, CD31, and von Willebrand factor but not CD45. Locally transplanted AT cells survived and participated in capillary networks in the ischemic tissues of immunodeficient nude rats in vivo. AT cells thus had multiple endothelial phenotypes and were defined as a major population of EPCs. Furthermore, laser Doppler and immunohistochemical analyses revealed that EPC transplantation quantitatively augmented neovascularization and blood flow in the ischemic hindlimb. In conclusion, umbilical cord blood is a valuable source of EPCs, and transplantation of cord blood-derived EPCs represents a promising strategy for modulating postnatal neovascularization.
\end{abstract}

J. Clin. Invest. 105:1527-1536 (2000).

\section{Introduction}

Therapeutic neovascularization is an important strategy to salvage tissue from critical ischemia (1-5). Neovascular formation in adults has been considered to result exclusively from the proliferation, migration, and remodeling of preexisting mature endothelial cells (ECs), a process referred to as angiogenesis (6-7). In contrast, vasculogenesis, a term defined as the formation of blood vessels from endothelial precursor cells (EPCs) or angioblasts during embryogenesis (8-11), begins as formation of blood islands that comprise EPCs at the periphery and hematopoietic stem cells (HSCs) at the center (12). Because EPCs and HSCs share several cell-surface antigens including KDR, Tie2/Tek, and CD34, these premature cells should derive from common mesodermal precursor cells (i.e., hemangioblasts) (12-14). Asahara et al. (15) recently discovered that peripheral blood of adult species contains progenitor ECs that presumably derived from CD34positive mononuclear cells $\left(\mathrm{MNC}^{\mathrm{CD} 34+}\right)$ and that the transfused EPCs participated in neovascular formation in mature immunodeficient animals. However, little is known as to whether in vivo transplantation of EPCs quantitatively augments neovascular formation in ischemic tissue in adults, a strategy consistent with "therapeutic vasculogenesis" (16).
Human umbilical cord blood has been shown to contain a large number of hematopoietic colony-forming cells (17). In contrast to adult bone marrow-derived HSCs, cord blood progenitors have distinctive proliferative advantages, including the capacity to form a greater number of colonies, a higher cell-cycle rate, and a longer telomere (18-20). All of these properties should favor the growth of the cord blood progenitors compared with adult peripheral blood or bone marrow progenitors (21-25). In addition, cord blood can be obtained noninvasively, in contrast to invasive bone marrow isolation. Accordingly, we tested our hypothesis that (a) human cord blood may be an alternative and novel source for isolating EPCs, and that (b) transplantation of cord blood-derived EPCs may participate in and effectively augment postnatal neovascularization in vivo.

\section{Methods}

Human umbilical cord blood. Human umbilical cord blood samples $(50-140 \mathrm{~mL}$ each; $n=102)$ were collected in sterile blood packs (SC-200; Terumo Corp., Tokyo, Japan) containing citrate-dextrose solution as the anticoagulant. Written informed consent was obtained from all mothers before labor and delivery. Protocols for sampling human umbilical cord blood were approved by the Institutional Review Board. 
Enumeration of $\mathrm{MNC}^{\mathrm{CD} 34+}$. Enumeration of $\mathrm{MNC}^{\mathrm{CD} 34+}$ in whole blood samples $(50 \mu \mathrm{L})$ of cord blood and adult peripheral blood was performed by flow cytometry (26). The method was a two-tube assay. The first tube contained (a) an FITC-conjugated nucleic acid binding dye to identify nucleated cells; (b) anti-CD45 mAb with peridinin chlorophyll protein (PerCP) to discriminate progenitor cells from mature lymphoid, neutrophil, erythroid, and monocytic cells; (c) a mouse IgG 1 conjugated with phycoerythrin (PE) to rule out nonspecific staining; and (d) a known number of fluorescent beads to determine absolute counts of cells. In the second tube, the IgG $\mathrm{I}_{1} \mathrm{PE}$ was replaced by an anti-CD34 $\mathrm{mAb}$ with PE (8G12; Becton Dickinson, San Jose, California, USA) to identify MNCCD34+.

Isolation of $\mathrm{MNC}^{\mathrm{CD} 34+}$. MNCs were isolated from umbilical cord blood or adult peripheral blood using the Histopaque-density centrifugation method (15). The MNC layer was collected, washed twice with $1 \mathrm{mM}$ EDTA in PBS, and suspended in degassed PBS with $0.5 \%$ BSA and 2 mM EDTA. $\mathrm{MNC}^{\mathrm{CD} 34+}$ were separated from 1-1.5 $\times 10^{8}$ MNCs by a magnetic bead separation method (MACS; Miltenyi Biotec, Gladbach, Germany). In brief, $\mathrm{MNC}^{\mathrm{CD} 34+}$ were labeled with a hapten-conjugated $\mathrm{mAb}$ against human CD34 (clone QBEnd/10), followed by an anti-hapten $\mathrm{Ab}$ coupled with microbeads. The bead-positive cells ( $\mathrm{MNC}^{\mathrm{CD} 34+}$ ) were enriched on positive-selection columns set in a magnetic field. Flow-cytometric analysis of purified cells using a different clone (8G12) of FITCconjugated anti-CD34 $\mathrm{mAb}$ showed that $79 \%( \pm 5 \%)$ of the selected cells were positive for CD34.

Cell culture. The medium used for cell culture experiments was Medium-199 supplemented with 20\% FBS, bovine pituitary extract as an EC growth supplement, heparin $(100 \mu \mathrm{g} / \mathrm{mL})$, and antibiotics (standard medium; Life Technologies, Grand Island, New York, USA). $\mathrm{MNC}^{\mathrm{CD} 34+}, \mathrm{MNC}^{\mathrm{CD} 34-}$, or total MNCs were cultured on human fibronectin-coated plastic plates (BIOCOAT; Becton-Dickinson). At days 4, 8, and 12 of culture, medium was replaced. The numbers of spindleshaped and attaching (AT) cells and cell clusters were counted under a phase-contrast microscope at days 7 and 14 of culture. Five randomly selected microscopic fields were evaluated, and mean numbers of AT cells and cell clusters were calculated in each cord blood or adult peripheral blood sample. The numbers of developed AT cells and cell clusters (per square millimeter) were compared between adult peripheral blood and cord blood. In some experiments, either selected $\mathrm{MNC}^{\mathrm{CD} 34+}$ or $\mathrm{MNC}^{\mathrm{CD} 34-}$ were labeled with a green fluorescent marker, PKH2-GL (Sigma Chemical Co., St. Louis, Missouri, USA), as described previously (27), and these labeled cells were cocultured with their unlabeled counterpart at a cell count ratio of 1:100 $\left(\mathrm{MNC}^{\mathrm{CD} 34+} / \mathrm{MNC}^{\mathrm{CD} 34-}\right)$. Human umbilical vein ECs (HUVECs) were obtained from the American Type Culture Collection (Rockville, Maryland, USA) and were cultured in the standard medium. HUVECs in passages between 3 and 18 were used.
Immunocytochemistry. Spindle-shaped and AT cells at day 7 of culture were subjected to immunocytochemistry to analyze the expression of von Willebrand factor (vWF), KDR/Flk-1, CD31, and ecNOS. In brief, cells were grown on chamber slides and fixed with cold methanol. Endogenous peroxidase was inactivated with $3 \%$ hydrogen peroxide. Nonspecific $\mathrm{mAb}$ binding was blocked by incubation with $10 \%$ horse serum. Primary mAb's directed against human vWF (clone F8/86; DAKO, Glostrup, Denmark), KDR (clone A-3; Santa Cruz Biotechnology Inc., Santa Cruz, California, USA), CD31 (JC/70A; DAKO) or ecNOS (clone 3; Transduction Laboratories, Lexington, Kentucky, USA) were then applied. These $m A b$ 's were all mouse $\operatorname{IgG}_{1}$, and thus negative control slides were incubated with appropriate dilution of MOPC-21 (Sigma), a nonimmune mouse $\operatorname{IgG}_{1}$. After two washes with PBS, a biotinylated horse anti-mouse $\mathrm{IgG}_{1} \mathrm{Ab}$ was applied, which was followed by the avidin-biotin immunoperoxidase treatment (Vector Laboratories, Burlingame, California, USA). To visualize the final immunoreaction products, 3-amino-9-ethylcarbazole (Histofine; Nichirei, Tokyo, Japan) was used.

Flow cytometry. Cord blood-derived AT cells at day 7 of culture $(n=8)$ were subjected to flow cytometric analysis to examine surface expression of KDR, VE-cadherin, CD31, CD34, and CD45. AT cells were isolated from culture plates at day 7 of culture by incubation using PBS with $1 \mathrm{mM}$ EDTA ( $\mathrm{pH}$ 7.4) for 15 minutes at $37^{\circ} \mathrm{C}$. Cells were fixed in $1 \%$ paraformaldehyde for 10 minutes at $4^{\circ} \mathrm{C}$. Aliquots of cells were then stained with primary mAb's directed to KDR/Flk-1 (clone KDR-1; Sigma Chemical Co.), VE-cadherin (clone BV6; Chemicon International, Temecula, California, USA), CD31 (clone L133.1), CD34 (clone 8G12), and a common leukocyte antigen, CD45 (clone 2D1; Becton-Dickinson). All mAb's recognize the extracellular domain of each molecule. After staining, cells were fixed with $1 \%$ paraformaldehyde and analyzed by flow cytometry.

Cellular uptake of acetylated LDL. We investigated incorporation of acetylated LDL (Ac-LDL) by AT cells, one of the characteristic features of cells in endothelial lin-

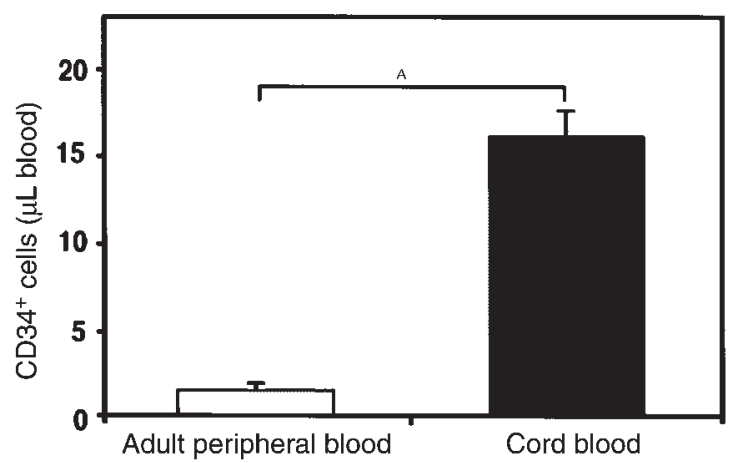

Figure 1

Enumeration of $\mathrm{MNC}^{\mathrm{CD} 34+}$ in umbilical cord blood and adult human peripheral blood. Umbilical cord blood contained 10-fold excess of $\mathrm{MNC}^{\mathrm{CD} 34+}$ compared with adult human peripheral blood. ${ }^{\mathrm{A} P}<0.001$. 


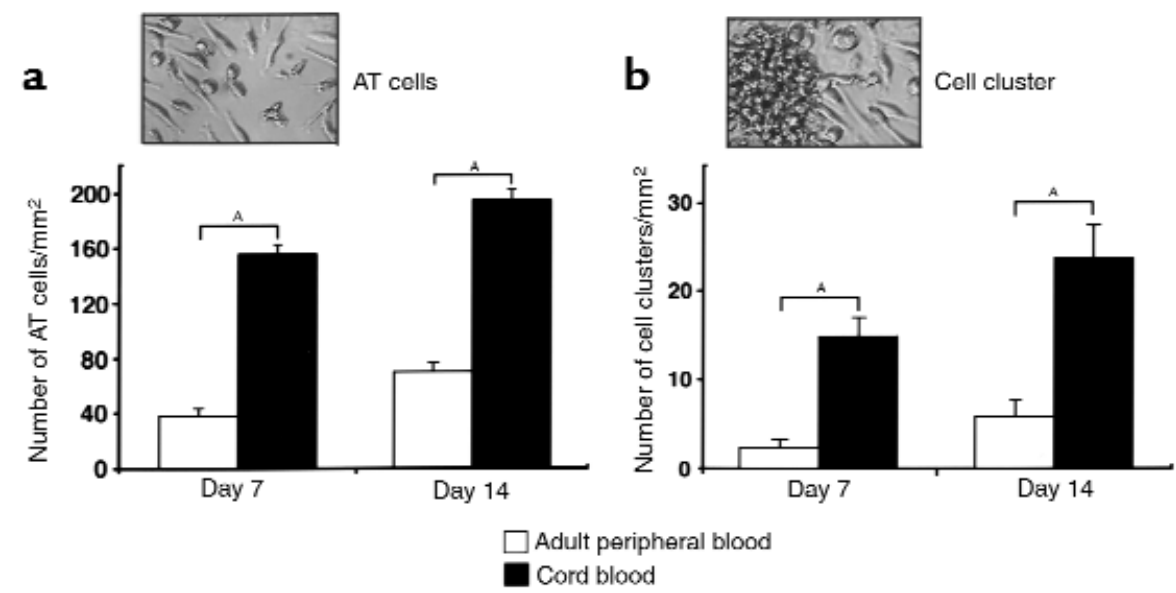

Figure 2

Comparison of numbers of AT cells and cell clusters between umbilical cord blood and adult human peripheral blood. Greater numbers of AT cells $(\mathbf{a})$ and cell clusters $(\mathbf{b})$ developed from similar numbers of MNCs from cord blood $(n=10)$ than from adult peripheral blood $(n=$ 7) examined at both days 7 and 14 of culture. ${ }^{A} P<0.001$. Representative photomicrographs of AT cells and a cell cluster developed from cord blood MNCs are shown at the top of each panel.

eage $(15,28,29)$. AT cells cultured on fibronectin were incubated in medium containing $15 \mu \mathrm{g} / \mathrm{mL}$ DiI-labeled Ac-LDL (DiI-Ac-LDL; Molecular Probes, Eugene, Oregon, USA) for 24 hours at $37^{\circ} \mathrm{C}$. Cells were then examined under a fluorescence microscope.

Detection of nitric oxide formation. Intracellular nitric oxide (NO) formation was analyzed using diaminofluorescein-2 diacetate (DAF-2 DA; Daiichi, Tokyo, Japan), a membrane-permeable NO-specific fluorescence indicator (30). When DAF-2 DA reacts with free $\mathrm{NO}$, the compound yields the green-fluorescent triazole (lower detection limit of $\mathrm{NO}=5 \mathrm{nM}$ ) (30). AT cells were gently washed twice with $\mathrm{Ca}^{2+}$-free PBS, and then bathed in Krebs-Henseleit buffer containing L-arginine $(1 \mathrm{mM})$ and DAF-2 DA $(10 \mu \mathrm{M})$. Cells were incubated at $37^{\circ} \mathrm{C}$ for an additional 15 minutes, and recombinant human $\operatorname{VEGF}_{165}(50 \mathrm{ng} / \mathrm{mL})$ (Genzyme Pharmaceuticals, Cambridge, Massachusetts, USA) was added to the wells. NO formation in the cells, as detected by nitrosylated DAF-2 DA, was examined under a fluorescence microscope.

In vitro angiogenesis model: endothelial network formation in basement matrix gel. At day 7 of culture, cord blood-derived AT cells were isolated from culture plates by incubating them with $1 \mathrm{mM}$ EDTA in PBS for 15 minutes at $37^{\circ} \mathrm{C}$. Cells were labeled with PKH2-GL and cocultured with unlabeled HUVECs on basement membrane matrix gel (Matrigel; Becton-Dickinson). At day 3 to day 7 of culture, formation of angiogenesis-like EC networks and incorporation of the fluorescentlabeled AT cells into the EC networks were examined under a fluorescence microscope.

In vivo angiogenesis model: unilateral hindlimb ischemia in immunodeficient nude rats. A rat model of surgically induced hindlimb ischemia was employed to determine whether transplanted cord blood-derived AT cells participated in postnatal neovascularization in vivo. For transplantation of human cells, immunodeficient
(F344/N rnu/rnu) nude rats were chosen to avoid graftversus-host disease. In the first series of experiments, eight male nude rats were subjected to severe limb ischemia by resection of the left femoral artery and vein under anesthesia with sodium pentobarbital $(50 \mathrm{mg} / \mathrm{kg}$, intraperitoneally). Rats were then transplanted with fluorescence-labeled AT cells $\left(3 \times 10^{5}\right.$ cells/rat $)$ intramuscularly in the ischemic thigh area using a 26-gauge needle. Two weeks after surgery, the animals were sacrificed with an overdose of pentobarbital. Ischemic skeletal muscles were isolated, embedded in OCT compound (Tissue-Tek; Miles Inc., Eckhart, Indiana, USA), and snap-frozen in liquid nitrogen. Multiple $6-\mu \mathrm{m}$ frozen sections were cut from each specimen so that the muscle fibers were oriented transversely. Fluorescence microscopy was used to detect transplanted labeled AT cells. In adjacent sections, alkaline phosphatase staining was performed using an indoxyl-tetrazolium method to detect viable capillary ECs as described previously (31). All animal protocols were approved by the Institutional Animal Care and Use Committee.

Efficacy of AT cell transplantation for postnatal neovascularization in vivo. We investigated whether local transplantation of cord blood-derived AT cells or adult peripheral blood-derived AT cells augmented neovascular formation in ischemic hindlimbs of nude rats (i.e., therapeutic vasculogenesis). Twenty-one additional nude rats were subjected to unilateral hindlimb ischemia. At day 3 after induction of limb ischemia, rats were injected in their ischemic adductor muscle area with either adult peripheral blood-derived AT cells $\left(3 \times 10^{6}\right.$ cells/animal, $\left.n=6\right)$, cord blood-derived AT cells $\left(3 \times 10^{6}\right.$ cells/animal, $\left.n=8\right)$, or saline (as a control, $n=7)$. Regional blood perfusion and histological capillary density were evaluated thereafter.

Laser Doppler perfusion image of the bindlimb blood flow. We measured the ratio of the ischemic (left)/normal (right) limb blood flow using a laser Doppler perfusion 
image (LDPI) analyzer (Moor Instruments, Devon, United Kingdom) (32). When laser scanning is performed, moving blood cells shift the frequency of the laser light according to the Doppler principle. Change in the frequency is displayed as a color-coded image representing blood flow (i.e., blood cell movement). Low to no flow is displayed as dark blue, whereas high flow is displayed as red to white. Before initiating laser scanning, rats were placed on a heating plate kept at $37^{\circ} \mathrm{C}$ to minimize data variations due to body temperature. At predetermined time points (before and on postoperative days 7,14 , and 21 ), we performed two consecutive scans over the same region of interest (legs and feet) in each animal and found essentially no difference between the two scans. After scanning them twice, the stored images were subjected to computer-assisted quantification of blood flow, and the average flow of the ischemic and nonischemic feet were calculated. To minimize data variables due to ambient light and temperature, the LDPI index was expressed as the ratio of left (ischemic) to right (nonischemic) limb blood flow.

Histological capillary density. Capillary densities of both ischemic and nonischemic skeletal muscle tissues were analyzed for specific evidence of vascularity at the level of the microcirculation. Ischemic and nonischemic hindlimb skeletal muscles were isolated and fixed in methanol overnight. Tissues were then embedded in paraffin and 5- $\mu \mathrm{m}$-thick sections were prepared. Anti-vWF mAb (clone F8/86) was used to identify ECs. Ten microscopic fields from three different sections from each animal were counted, and capillary density was expressed as the number of capillaries per square millimeter.

Statistics. All values are presented as the means plus or minus SE. All data were subjected to ANOVA followed by the Fisher's analysis for comparison between two means. Probabilities less than 0.05 were considered to be statistically significant.

\section{Results}

Enumeration of $M N C^{C D 34+}$. Flow cytometric analysis revealed that umbilical cord blood $(n=12)$ contained a 10-fold excess of $\mathrm{MNC}^{\mathrm{CD} 34+}$ when compared with adult peripheral blood $(n=9 ; P<0.001)$ (Figure 1 ).

Development of cell clusters and sprouting of spindle-shaped and AT cells from MNCs. When cord blood MNCs $(n=20)$ were cultured on fibronectin, a number of cell clusters appeared within 48 hours, and numerous spindle-shaped and AT cells sprouted from the edge of the cell clusters at day 3 of culture (Figure 2). The spindle-shaped AT cells were observed as either isolated cells or linear cord-like structures. These morphological structures resembled those of endothelial progenitor cells developed from adult human peripheral blood, which we reported recently (15). Much greater numbers of AT cells and cell clusters developed from MNCs of cord blood $(n=10)$ than from the same amount of MNCs of adult peripheral blood $(n=7 ; P<0.001)$ (Figure 2).
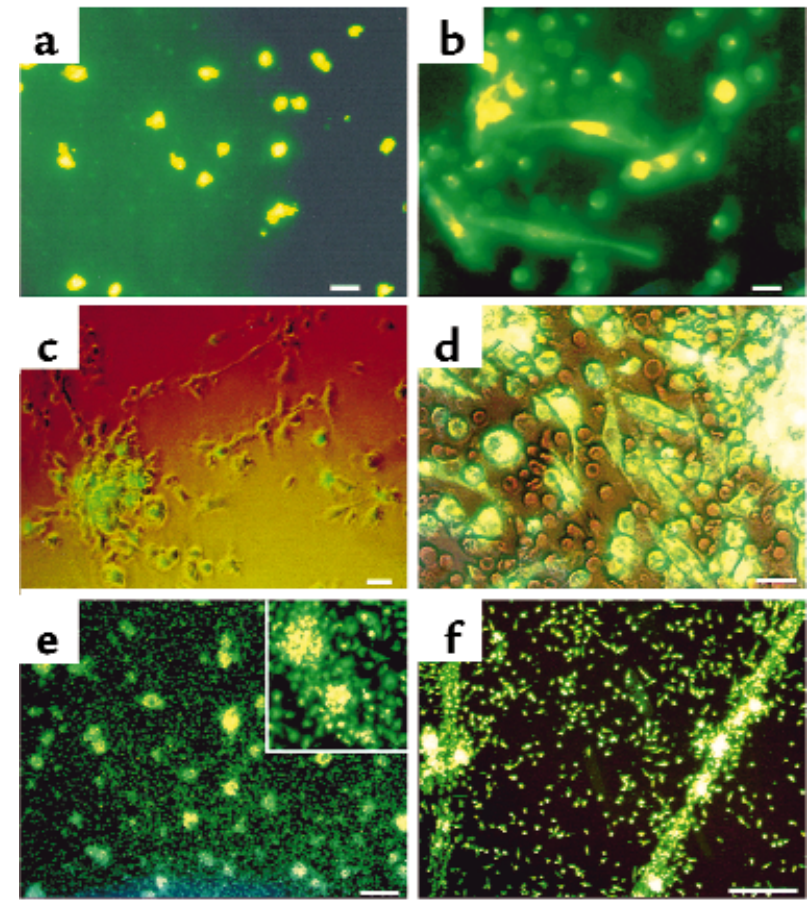

\section{g}
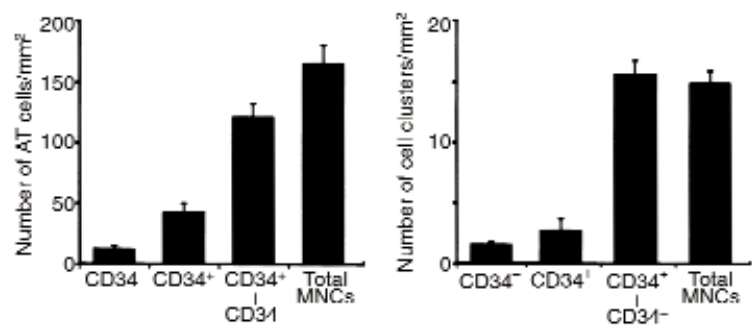

Figure 3

Development of spindle-shaped and AT cells, and cord-like structures from $\mathrm{MNCCD}^{\mathrm{CD} 3+}$. (a) When labeled $\mathrm{MNCCD}^{\mathrm{C} 3-}$ alone were plated on fibronectin, most of the cells did not attach to fibronectin and had a round shape. (b) When labeled MNCCD34+ alone were plated on fibronectin, only limited numbers of cell clusters and AT cells appeared within 7 days. (c) When green fluorescence-labeled MNCCD34- were coplated with unlabeled $\mathrm{MNC}^{\mathrm{CD} 34+}$, fluorescence-positive MNCCD34- participated in cell cluster formation, but they were located only at the center of the clusters. AT cells and cord-like structures were mostly fluorescence negative. (d) Conversely, when fluorescence-labeled $\mathrm{MNC}^{\mathrm{CD} 34+}$ were coplated with unlabeled $\mathrm{MNC}^{\mathrm{CD} 34-}$, the AT cells, cell clusters, and cord-like structures were mostly positive for the fluorescence. (e, f) From coculture of fluorescence-labeled $\mathrm{MNCCD}^{\mathrm{C} 4+}$ and unlabeled MNC ${ }^{\mathrm{CD} 34-}$, numerous fluorescence-positive cell clusters developed, and some of the cell clusters connected together to form linear cord-like structures. Bars: $20 \mu \mathrm{m}$ in $\mathbf{a}-\mathbf{d}, 100 \mu \mathrm{m}$ in $\mathbf{e}$ and $\mathbf{f}$. (g) Bar graphs showing the numbers of AT cells and cell clusters developed from four different conditions of cell culture $\left(1,000\right.$ cells $\left./ \mathrm{mm}^{2}\right)$. A limited number of AT cells and cell clusters appeared from $\mathrm{MNCCD}^{\mathrm{C}} 34^{+}$alone. The numbers of developed AT cells and cell clusters were greatly enhanced by coculture of $\mathrm{MNC}^{\mathrm{CD} 34+}$ with $\mathrm{MNC}^{\mathrm{CD} 34-}$ when compared with culture of $\mathrm{MNCCD}^{\mathrm{C} 3+}$ alone. The numbers of AT cells and cell clusters derived from coculture of $\mathrm{MNC}^{\mathrm{CD} 34+}$ and $\mathrm{MNCCD}^{\mathrm{C} 34}$ were comparable to those from total MNCs. 
$M C^{C D 34+}$ developed into AT cells and cord-like structures. Because CD34 is expressed on both hematopoietic and endothelial cell lineages, we tested whether selected $\mathrm{MNC}^{\mathrm{CD} 34+}$ could give rise to AT cells. $\mathrm{MNC}^{\mathrm{CD} 34+}$ were separated from cord blood MNC by a magnetic-bead method. Flow cytometric analysis revealed that $79 \%$ $( \pm 5 \%)$ of the selected $\mathrm{MNC}^{\mathrm{CD} 34+}$ expressed CD34 $(n=12)$, whereas less than $0.5 \%$ of the remaining cells (MNCCD34-) expressed CD34. In the first set of experiments, we labeled MNCCD34- with a green fluorescent marker and cultured them on fibronectin. From the labeled MNCCD34- alone, most of the cells did not attach to the plates (Figure 3a) when examined for up to 4 weeks. We next labeled $\mathrm{MNC}^{\mathrm{CD} 34+}$ with the fluorescence marker. From labeled $\mathrm{MNC}^{\mathrm{CD} 34+}$ alone, limited numbers of cell clusters appeared and AT cells were observed only sporadically (Figure $3 \mathrm{~b}$ ). When fluorescencelabeled $\mathrm{MNC}^{\mathrm{CD} 34-}$ were cocultured with unlabeled $\mathrm{MNC}^{\mathrm{CD} 34+}$, AT cells and cord-like structures were mostly negative for the fluorescence (Figure 3c). However, labeled $\mathrm{MNC}^{\mathrm{CD} 34-}$ could be identified at the center of the cell clusters (Figure 3c). Conversely, when MNC $\mathrm{CD}^{\mathrm{CD}}+$ were fluorescence-labeled and cocultured with unlabeled MNCCD34-, AT cells, cell clusters, and cord-like structures were fluorescence positive. These fluorescence-trace experiments suggest that a population of $\mathrm{MNC}^{\mathrm{CD} 34+}$ gave rise predominantly to AT cells, cell clusters, and cord-like structures. Cell clusters comprised round cells at the center and spindle-shaped AT cells at the periphery (Figure 3,c-e). These morphological characteristics and cellular organization might resemble the structure of blood islands observed in dissociated quail epiblast cultures that gave rise to primary vascular networks (12). The cell clusters occasionally connected with each other to form linear cord-like structures (Figure 3f) that might mimic the blood-island fusion and forma- tion of a primordial vascular network in the embryo (8-10). Interestingly, coculture of $\mathrm{MNC}^{\mathrm{CD} 34+}$ with MNCCD34- much increased the number of AT cells (threefold) and cell clusters (sixfold), compared with culture of $\mathrm{MNC}^{\mathrm{CD} 34+}$ alone (Figure $3 \mathrm{~g}$ ), indicating that the development of AT cells seems to require certain interactions between $\mathrm{MNC}^{\mathrm{CD} 34+}$ and $\mathrm{MNC}^{\mathrm{CD} 34-}$.

AT cells had multiple endothelial phenotypes. To examine whether AT cells have endothelial phenotypes, AT cells were subjected to immunocytochemistry. AT cells were positively stained (> 98\%) for vWF, CD31, and KDR (figures not shown). In addition, more than $95 \%$ of the AT cells at day 7 of culture took up DiI-ac-LDL (Figure $4 a)$, one of the characteristic functions of ECs (28). AT cells at day 7 of culture were positively stained for endothelial constitutive NO synthase (ecNOS) (Figure $4 \mathrm{~b})$. Because VEGF mediates endothelial NO release through the KDR/Flk-1 receptor tyrosine kinase (33, 34 ), and because the AT cells expressed KDR in the present study, we examined whether recombinant human $V_{E G F} F_{165}\left(\mathrm{hVEGF}_{165}\right)$ stimulated NO release from AT cells. Addition of hVEGF $165(50 \mathrm{ng} / \mathrm{mL})$ into cell culture plates containing AT cells in Krebs-Henseleit buffer greatly enhanced NO release as assessed by a fluorescent NO indicator, DAF-2 DA (Figure 4, c and d). The evidence of hVEGF-induced NO release confirmed that the AT cells can functionally respond to VEGF through activation of its receptor tyrosine kinase followed by activation of the ecNOS (35-38). These findings suggest that AT cells express several endothelial markers and have endothelial functions, and thus AT cells have endothelial phenotypes.

We further performed flow cytometric analyses of AT cells at day 7 of culture to examine the expression of KDR, VE-cadherin, CD34, and CD31, as markers for endothelial lineage, and CD45 as a common leukocyte
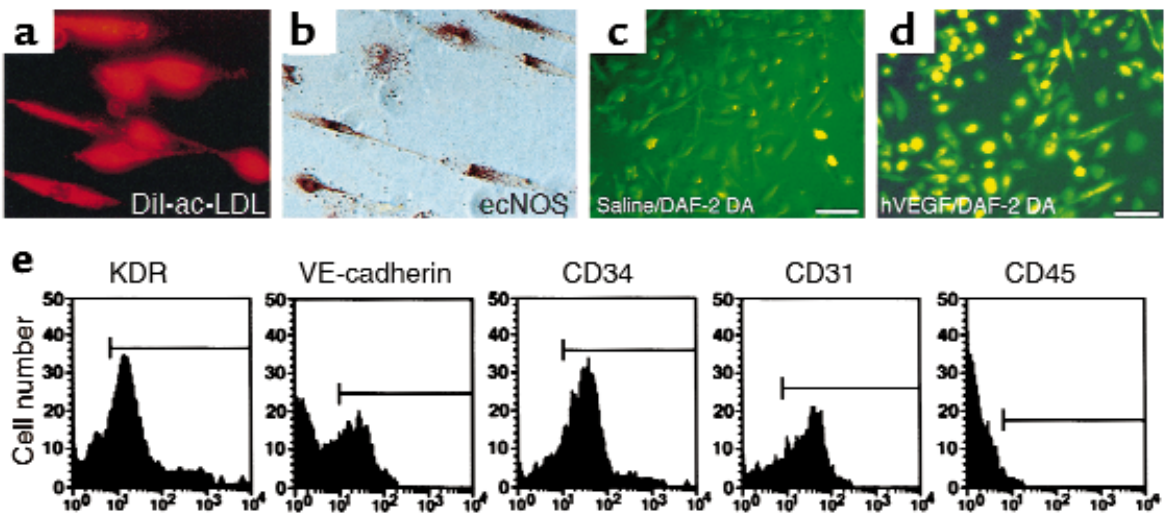

VE-cadherin
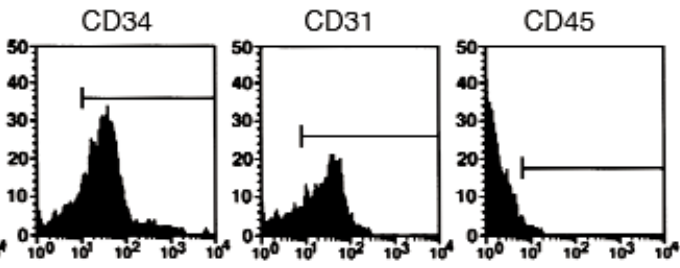

Fluorescence intensity

\section{Figure 4}

AT cells had EC-like functions and expressed cell surface markers in vitro. At day 7 of culture (a) AT cells took up Dil-ac-LDL. (b) AT cells were positive for endothelial constitutive NO synthase (ecNOS) immunostaining. (c and d) Incubating AT cells with DAF-2 DA, a NO-specific fluorescence indicator, showed that $\mathrm{hVEGF}_{165}(50 \mathrm{ng} / \mathrm{mL})(\mathbf{d})$, but not saline $(\mathbf{c})$, induced NO release from AT cells. Bars, $50 \mu \mathrm{m}$ in c and d. (e) Flow cytometric analyses of AT cells at day 7 of culture $(n=8)$. Horizontal bars indicate positive antigen gates as determined by an isotype-matched IgG control study. AT cells were positive for KDR (52 $\pm 5 \%$ ), VE-cadherin (62 $\pm 2 \%)$, CD34 (62 $\pm 7 \%)$, and CD31 (57 \pm $9 \%)$, but not for CD45 ( $\pm \pm 3 \%)$. Data are percentage of positive cells. 

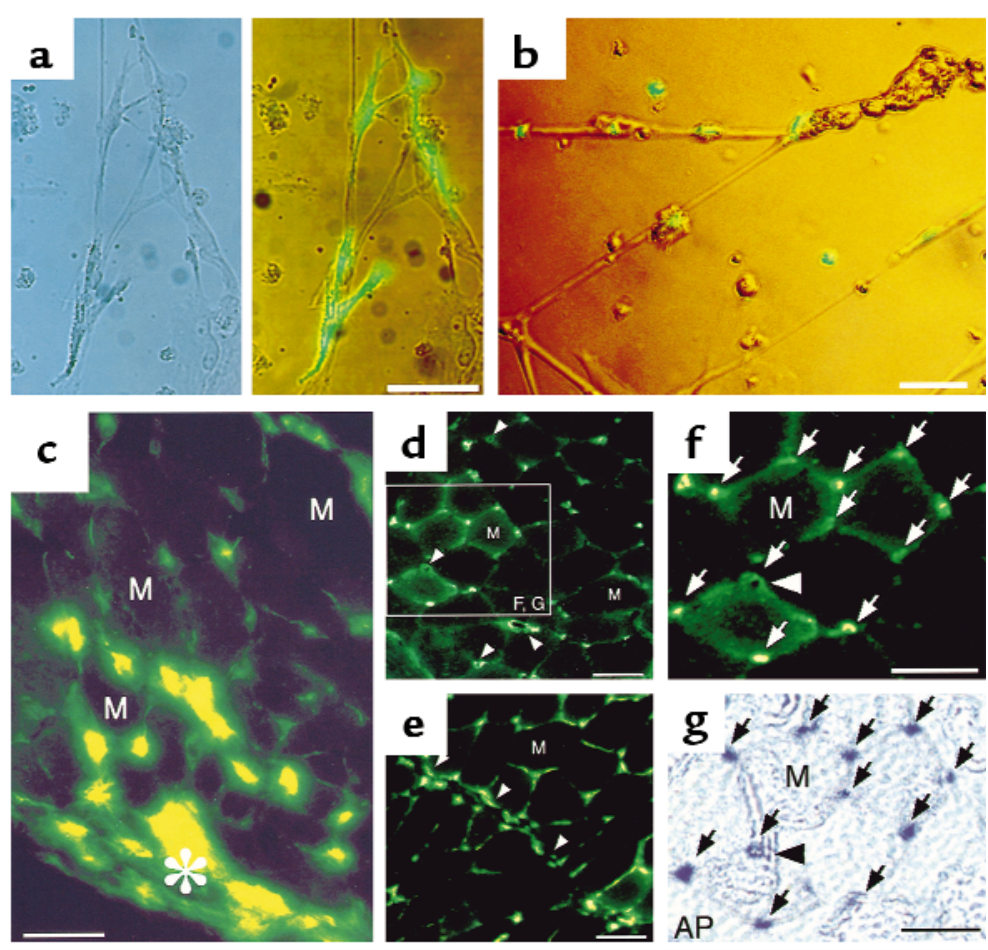

Figure 5

Participation of cord blood-derived AT cells in neovascularization in vitro and in vivo. (a) The phase-contrast (left) and fluorescent (right) photomicrographs at the same microscopic field are shown. Green fluorescencelabeled AT cells contacted unlabeled HUVECs on matrix gel within 24 hours of coculture. (b) The labeled AT cells were incorporated into the EC network formed on the basement matrix gel within 3 days after coculture. (c-g) Frozen sections of tissues harvested from the ischemic hindlimb of nude rats 2 weeks after transplantation of fluorescence-labeled cord blood-derived AT cells. The labeled AT cells were sprouting from a place near the cell injection site indicated by * (c). AT cells further migrated into the interstitial regions among preserved skeletal myocytes (indicated by M). Numerous labeled AT cells were incorporated into and formed capillary-like networks (arrows in $\mathbf{e}$ and $\mathbf{g}$ ), as well as tubular structures with a round lumen (arrowheads $\mathbf{d}-\mathbf{g}$ ). (f) Green fluorescence signals indicate localization of transplanted AT cells forming numerous capillary-like networks (arrows). (g) The phase-contrast photomicrograph of an adjacent section histochemically stained with alkaline phosphatase (AP) shows viable capillary ECs (dark blue colors) at identical locations of the incorporated AT cells seen in $\mathbf{f}$. Bars, $50 \mu \mathrm{m}$. antigen. AT cells ( $n=8$ in cord blood) were stained positive for KDR, VE-cadherin, CD34, and CD31 (Figure 4e), whereas only few AT cells expressed CD45, a common leukocyte antigen (Figure 4e).

Cord blood-derived AT cells participated in EC networks in vitro. To examine whether cord blood-derived AT cells participated in endothelial network formation in vitro, AT cells were collected at day 7 of culture, fluorescence labeled, and cocultured with unlabeled HUVECs on basement membrane matrix gel. Within 24 hours of coculture, the labeled AT cells started to contact HUVECs (Figure 5a). Within 3 days, incorporation of fluorescence-labeled AT cells was identified within the HUVEC networks (Figure 5b). However, when AT cells alone were cultured on the matrix gel, EC networks were poorly organized (figure not shown). These results suggest that AT cells actively form endothelial networks when they were cocultured with mature ECs on matrix gel in vitro, indicating that interaction between AT cells and mature ECs may facilitate EC-directed differentiation of AT cells in vitro.

Transplanted cord blood-derived AT cells participated in neovascularization in the ischemic hindlimb of immunodeficient nude rats in vivo. We examined whether transplanted cord blood-derived AT cells participated in postnatal neovascularization in immunodeficient adult animals in vivo. AT cells were isolated at day 7 of culture and were fluorescence labeled. Unilateral hindlimb ischemia was surgically induced in the nude rats. Three days after surgery, rats $(n=8)$ were injected with fluorescence-labeled cord blood-derived AT cells $\left(3 \times 10^{5}\right.$ cells/animal $)$ in the ischemic thigh skeletal muscles. At day 14 after induction of limb ischemia, frozen tissue sections were prepared from the ischemic tissues. Fluorescence microscopic examination revealed that numerous labeled AT cells were incorporated and arranged into EC capillaries among the preserved skeletal myocytes in the ischemic hindlimbs (Figure 5, c-f). Moreover, transplanted AT cells often formed tubular structures with a round lumen (Figure 5, d and e). When adjacent sections were stained for alkaline phosphatase to identify viable ECs (23), capillary ECs were detected at the exact same locations where fluorescence-positive cells were identified (Figure 5, f and g). Thus, transplanted AT cells survived (stained positive for alkaline phosphatase) and were incorporated into the capillary networks in the ischemic hindlimb of nude rats in vivo. Because cord blood MNC-derived AT cells expressed multiple endothelial lineage markers and functions ( $\mathrm{NO}$ release and ac-LDL incorporation) and because AT cells participated in neovascular formation both in vitro and in vivo, we defined AT cells as being a major population of EPCs that derived from cord blood.

Transplantation of EPCs quantitatively augmented ischemia-induced neovascularization in nude rats. Finally we examined whether in vivo transplantation of EPCs might quantitatively augment postnatal neovascularization in the ischemic hindlimb in nude rats. Unilateral (left) limb ischemia was created in 21 additional animals, and EPCs isolated at day 7 of culture were locally transplanted into the ischemic thigh muscle area $\left(3 \times 10^{5} \mathrm{EPCs} / \mathrm{rat}\right)$ at four different injection points. In these in vivo experiments, we also compared the efficacy of therapeutic neovascularization by EPC transplantation between adult human peripheral blood and cord blood. Serial LDPI analyses revealed significantly augmented ratios of the ischemic/nor- 
mal hindlimb blood flow in the EPC-transplanted groups compared with saline-treated controls at postoperative days 7, 14, and 21 (Figure 6, a and b). Transplantation of cord blood-derived EPCs or adult peripheral blood-derived EPCs augmented the ischemic/normal blood flow ratio almost equally (Figure 6 , a and b). At postoperative day 14, immunohistochemical analysis of vWF and histochemical staining for alkaline phosphatase in the ischemic tissues revealed a significant increase in the capillary density by transplantation of either cord blood or adult peripheral blood EPCs compared with the saline controls (Figure 6, c and d). The capillary densities did not statistically differ between the cord blood EPCand adult peripheral blood EPC-transplanted group. No significant difference in the capillary densities was observed among the three groups in the contralateral nonischemic right hindlimb (Figure $6 \mathrm{~d}$ ). Therefore, as long as similar amounts of EPCs $\left(3 \times 10^{5}\right.$ cells/animal $)$ from either origins (i.e., adult peripheral blood or cord blood) are transplanted, ischemia-induced neovascularization and blood flow increase seemed to be similarly augmented in vivo.

\section{Discussion}

Human umbilical cord blood contains a large amount of hematopoietic progenitors $(17,18)$. The present study demonstrates that angioblast-like EPCs can also be isolated from cord blood. In addition, greater numbers of EPCs developed from cord blood MNCs than from same amount of adult peripheral blood MNCs. Isolated EPCs were incorporated into HUVEC networks on matrix gel in culture, and transplanted EPCs participated in postnatal neovascularization in the ischemic hindlimb in vivo. Moreover, EPC transplantation quantitatively augmented ischemia-induced neovascularization and regional blood flow in immunodeficient animals in vivo.

Recently, EPCs have been identified in peripheral blood of adult humans and mature animals (15). EPCs may reside in the adult bone marrow and are mobilized

\section{a}

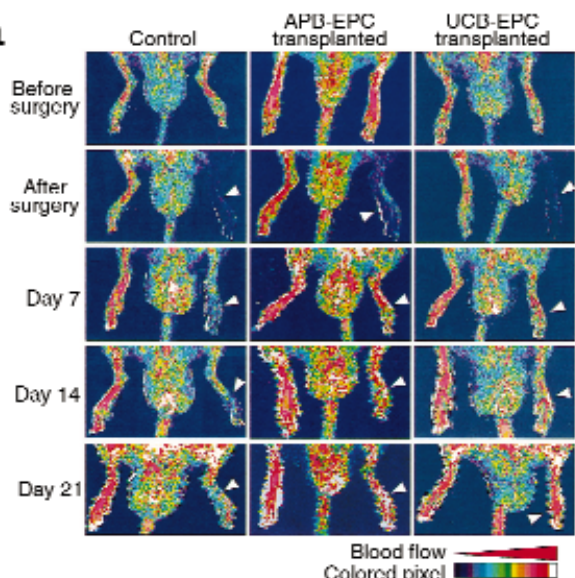

b

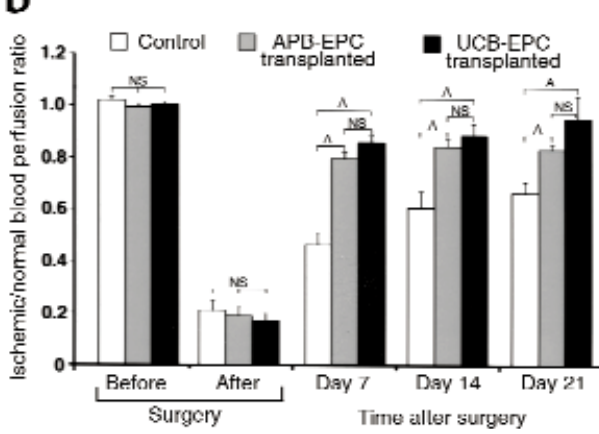

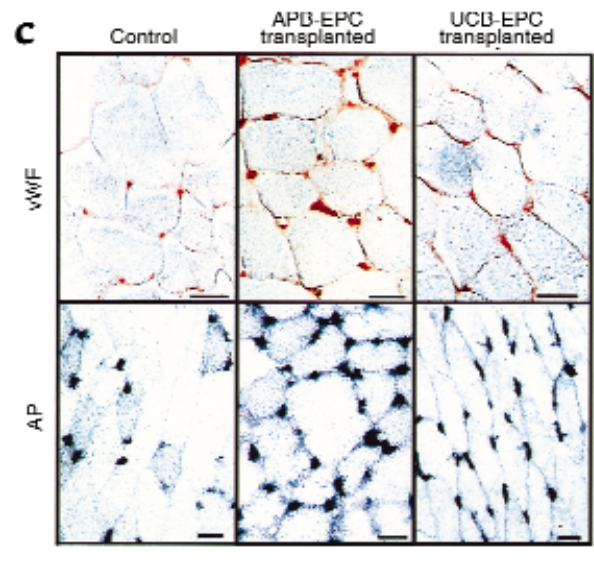

d

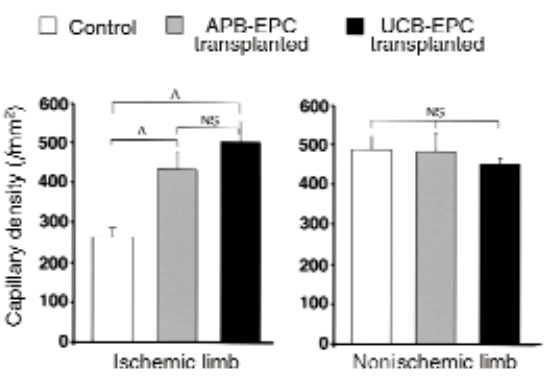

\section{Figure 6}

Augmented neovascularization by local transplantation of either umbilical cord blood-derived (UCB-derived) or adult peripheral blood-derived (APB-derived) EPCs in the ischemic hindlimb of immunodeficient nude rats. (a) Unilateral (left) hindlimb ischemia (arrowheads) was surgically induced in nude rats. Either APB-derived EPCs $\left(3 \times 10^{5}\right.$ cells/animal, $\left.n=6\right), \mathrm{UCB}$-derived EPCs $\left(3 \times 10^{5} \mathrm{cells} /\right.$ animal, $n=8$ ), or saline (control, $n=7$ ) were locally transplanted into the ischemic hindlimb at postoperative day 3 . Representative serial LDPI analyses showed progressive increases in blood flow in the ischemic hindlimb (red to white color) of rats injected with EPCs obtained from APB or UCB. In contrast, recovery of blood flow was retarded in the ischemic limb (blue to green color) in a control animal. (b) Computer-assisted quantitative analyses of hindlimb blood flow demonstrated significantly enhanced ischemic/normal limb blood perfusion ratios in rats injected with EPCs of either origin (APB or UCB) compared with controls. ${ }^{A} P<0.001$. (c) Capillary ECs were identified in the sections of ischemic tissues under a light microscope after immunohistochemical staining for vWF (brown reaction products) and alkaline phosphatase (AP) staining (blue reaction products). Light microscopy revealed increased capillary densities in rats receiving EPCs of either origin (APB or UCB) compared with controls. Bars, $50 \mu \mathrm{m}$. (d) Quantitative analyses showed increased capillary densities in the ischemic hindlimb tissues of the two EPC-transplanted groups as compared with control animals examined 14 days after induction of limb ischemia. ${ }^{A} P<0.001$. 
into the peripheral circulation in response to cytokines or tissue ischemia $(39,40)$. Umbilical cord blood has been used as an alternative source for isolation of HSCs (21-25). In contrast to adult bone marrow progenitors, cord blood progenitors have distinctive proliferative advantages (18-20). This property should favor the engraftment and growth of the cord blood progenitor cells. The present study demonstrates that EPCs can develop from cord blood $\mathrm{MNC}^{\mathrm{CD} 34+}$ in culture. Moreover, a greater number of EPCs and cell clusters developed from cord blood MNCs than from similar amount of adult peripheral blood MNCs (Figure 2), which was consistent with our flow-cytometric analysis showing that cord blood contained much greater amounts of $\mathrm{MNC}^{\mathrm{CD} 34+}$ than adult peripheral blood (Figure 1). Thus, umbilical cord blood seems to be a much more robust source for isolating not only HSCs but also EPCs.

Cell surface molecules such as CD34, KDR, Tie$2 /$ Tek, and VE-cadherin are expressed by ECs at the early stage of vasculogenesis (41-48). CD34 is also expressed by mature ECs in adults $(49,50)$. Similarly, HSCs express CD34, KDR, and Tie-2/Tek on their surface (51-53). CD34, however, is lost by HSCs as they differentiate into mature blood cells (54-56). In an early study, Rafii et al. demonstrated colonization of the flow surface of the titanium housing of left ventricular assist devices with $\mathrm{CD}^{+} 4^{+}$EC-like cells 6 months after the devices were removed (57). More recently, Shi et al. (58) showed that transplanted bone marrow-derived $\mathrm{CD}_{3} 4^{+}$cells participated in the endothelialization of impervious Dacron grafts in vivo. Therefore, we consider that the CD34 antigen is one of appropriate landmark molecules for isolation of putative EPCs from circulating blood. This strategy was also employed in our previous study (15).

To examine whether $\mathrm{MNC}^{\mathrm{CD} 34+}$ might develop into EPCs in vitro, we performed fluorescence-labeled cell trace experiments. When $\mathrm{MNC}^{\mathrm{CD} 34+}$ were fluorescence labeled and cocultured with unlabeled MNCCD34-, AT cells, cell clusters, and cord-like structures were mostly fluorescence positive, indicating that $\mathrm{MNC}^{\mathrm{CD} 34+}$ might predominantly differentiate into AT cells (Figure 3d). Interestingly, when $\mathrm{MNC}^{\mathrm{CD} 34+}$ and $\mathrm{MNC}^{\mathrm{CD} 34-}$ were remixed and coplated, a greater number of cell clusters and AT cells developed compared with cultures of $\mathrm{MNC}^{\mathrm{CD} 34+}$ alone (Figure 3g). Thus, $\mathrm{MNC}^{\mathrm{CD} 34+}$ seem to require either a cell-to-cell interaction with $\mathrm{MNC}^{\mathrm{CD} 34-}$ or exposure to soluble factors released from MNCCD34-, or both, to differentiate into AT cells in vitro. To further examine how $\mathrm{MNC}^{\mathrm{CD} 34-}$ interact with $\mathrm{MNC}^{\mathrm{CD} 34+}$ during AT cell development, $\mathrm{MNC}^{\mathrm{CD} 34-}$ were conversely fluorescence labeled and cocultured with unlabeled $\mathrm{MNC}^{\mathrm{CD} 34+}$. Although fluorescence-labeled MNCCD34participated in cell cluster formation, they were located only centrally in the clusters. AT cells at the edge of clusters and cord-like structures were mostly fluorescence negative (Figure 3c). These fluorescence-trace experiments suggest that (a) AT cells and cord-like structures likely developed from $\mathrm{MNC}^{\mathrm{CD} 34+}$ fraction and that (b)
MNCCD34- might support EPC differentiation from $\mathrm{MNC}^{\mathrm{CD} 34+}$ through an interaction with $\mathrm{MNC}^{\mathrm{CD} 34+}$. Because $\mathrm{MNC}^{\mathrm{CD} 34-}$ were located only in the center of the clusters (i.e., blood island-mimicking structures), some $\mathrm{MNC}^{\mathrm{CD} 34-}$ might play a role in hematopoietic reconstitution as reported recently (59).

Currently, there has been no exclusive marker for EPCs (60). Our present findings, however, suggest that AT cells sprouting from cell clusters are likely a major population of EPCs for several reasons. First, immunocytochemical and flow-cytometric analyses revealed that AT cells expressed multiple (not single) molecules such as KDR, CD31, vWF, CD34, and VE-cadherin, which are commonly used for identification of ECs (28, 41). In particular, KDR, CD34, and VE-cadherin have been used as markers for identification of progenitor ECs $(14,15,41)$. Second, AT cells expressed ecNOS protein and were able to release NO in response to VEGF. We showed previously that VEGF but not placenta growth factor, the ligand that exclusively binds to Flt1 , stimulated NO release in human ECs (33). Thus, the current evidence that VEGF stimulates NO release from AT cells confirms the presence of the functional VEGF-KDR-ecNOS pathway in AT cells. AT cells also took up ac-LDL. Thus, AT cells have multiple EC markers and functions. Third, AT cells sprouted from clusters of MNCs at a delayed time course (about 7 days) of culture in the present study. This outgrowth pattern of AT cells differs from that of cell outgrowth from mature ECs in culture. Fourth, fluorescence-labeled AT cells participated in an angiogenesis-like endothelial network formation with HUVECs on matrix gel in culture. Finally, transplanted AT cells formed capillarylike structures and tubular structures with a round lumen in the ischemic hindlimb tissues of nude rats in vivo. Based on these characteristics of AT cells, we defined AT cells as being a major population of EPCs in the present study. While this manuscript was in preparation, Peichev et al. reported that $\mathrm{MNC}^{\mathrm{KDR}+\mathrm{AC} 133+}$ within the fraction of $\mathrm{MNC}^{\mathrm{CD} 34+}$ represented putative EPCs in humans (61). Discovery of such identification markers for EPCs would further facilitate isolation and therapeutic use of EPCs in the future.

The presence of mature circulating ECs (CECs) is also possible. Solovey et al. (62), however, demonstrated that the number of CECs in peripheral blood was low (i.e., 2 to 4 cells $/ \mathrm{mL}$ of blood). Mutin et al. (63) also showed that the numbers of CECs were very few (almost zero) in healthy subjects, and ranged from 10 to 40 cells $/ \mathrm{mL}$ in patients with acute myocardial infarction. However, the numbers of EPC that outgrew from culture of cord blood-MNCs in the present study were much greater (i.e., 500 to 1,000 cells $/ \mathrm{mL}$ blood in our calculation) than numbers of CECs that reported previously $(62,63)$. Therefore, we consider that expanded outgrowth of EPCs from cord blood MNCs were not likely derived from CECs in the present study. Recently, Lin et al. (64), employing sex-mismatched bone marrow transplantation patients, also showed that delayed 
and expanded outgrowth of ECs from culture of adult peripheral blood should derive from circulating EPCs that originated from bone marrow. A possible reason of the more rapid outgrowth of EPCs from umbilical cord blood (about 7 days) in the present study compared with the study by Lin et al. (about 27 days) might be due to the fact that cord blood progenitors have proliferative advantages (e.g., longer telomeres and higher cell-cycle rate) compared with adult bone marrow progenitors $(18,19)$. Currently, it is unknown whether EPCs in the umbilical cord blood derived from fetal bone marrow. In fact, the umbilical cord is also a unique site of hematopoiesis and EC development. In the mouse embryo, for example, the endothelium of the proximal portion of the umbilical arteries displays a rounded morphology with clusters of $\mathrm{MNC}^{\mathrm{CD} 34+}$ bound to the luminal surface (65). This pattern may mimic that described for the paraaortic hematopoietic sites in the avian embryo (66). The umbilical cord itself may comprise sites in which both EPCs and HSCs develop and are released into the fetal circulation.

In the present study, EPCs were incorporated into HUVEC networks formed on basement matrix gel in vitro (Figure 5, a and b). Our in vivo study further indicated that transplanted cord blood-derived EPCs were incorporated into the capillary networks among the preserved skeletal myocytes in the ischemic limb of immunodeficient nude rats. The fact that transplanted cells were costained with alkaline phosphatase (Figure $5 \mathrm{~g}$ ) provides evidence that the transplanted EPCs survived in the ischemic tissues because positive alkaline phosphatase staining depends on vital enzymatic activity within capillary ECs (31). More importantly, both the LDPI and histological capillary density analyses showed that local transplantation of EPCs into the ischemic limb quantitatively augmented neovascular formation and blood flow in the ischemic limb in vivo. To our knowledge, this is the first study demonstrating that transplantation of EPCs quantitatively enhanced neovascularization in adult tissues, a notion consistent with therapeutic vasculogenesis (16).

It has been reported that cord blood is a much robust source for isolating HSCs compared with adult peripheral blood or bone marrow. We thus compared the numbers of developed AT cells (major population of EPCs) and cell clusters between the cord blood and adult peripheral blood. Greater numbers of AT cells and cell clusters developed from cultures of cord blood MNCs than from an equal amount of peripheral blood MNCs (Figure 2). Thus, umbilical cord blood seems to be a much more robust source for isolating EPCs than adult peripheral blood. We also compared the efficacy of therapeutic neovascularization in the ischemic limb of nude rats between cord blood EPC and peripheral blood EPC transplantations $\left(3 \times 10^{5}\right.$ cells/animal $)$. Transplantation of either cord blood EPCs or peripheral blood EPCs almost equally augmented the ischemic/normal blood flow ratios and capillary densities. These results suggest that the efficacy of thera- peutic neovascularization is comparable between cord blood and peripheral blood EPCs as long as similar amounts of EPCs are transplanted. Our results are consistent with a very recent study by Kalka et al. (67), who showed that transplantation of adult peripheral bloodderived EPCs significantly augmented ischemiainduced neovascularization in the hindlimb and promoted limb salvage in nude mice. However, considering the fact that cord blood is a much more robust source for EPCs than adult peripheral blood, cord blood may still be a novel source for EPCs. Moreover, cord blood can be isolated noninvasively compared with adult bone marrow isolation.

In summary, our findings suggest that umbilical cord blood is a precious source for isolating EPCs, and transplantation of EPCs significantly augmented postnatal neovascularization (16). While this manuscript was in preparation, Crisa et al. (68) also reported that transplanted cord blood progenitors sustained not only $\mathrm{T}$ lymphocytes but also induced angiogenesis in the implanted human thymus in the kidney of NOD/SCID mice. Our present findings further raise several important clinical implications. First, homologous or autologous transplantation of cord blood-derived EPCs may augment postnatal neovascularization in ischemic tissues (i.e., therapeutic vasculogenesis). Second, the fact that transplanted EPCs were incorporated into sites of neovascularization in adult tissues suggests a potential use of EPCs as cell vectors for gene delivery to angiogenic sites in vivo.

\section{Acknowledgments}

We thank K. Kimura, A. Shimizu, K. Moriyama, and M. Tsuru for expert technical assistance; J.M. Isner and T. Asahara for comments and continuous encouragement; S.T. Mendenhall for reading this manuscript. This work is supported by grants (11557058 and 11158220) from the Ministry of Education, Science, Sports and Culture of Japan, the Naito Foundation, the Ishibashi Foundation, and the Japan Heart Foundation (T. Murohara).

\footnotetext{
1. Yanagisawa-Miwa, A., et al. 1992. Salvage of infarct myocardium by angiogenic action of basic fibroblast growth factor. Science. 257:1401-1403.

2. Isner, J.M., et al. 1996. Clinical evidence of angiogenesis following arterial gene transfer of phVEGF ${ }_{165}$. Lancet. 348:370-374.

3. Pearlman, J.D., et al. 1995. Magnetic resonance mapping demonstrates benefits of VEGF-induced myocardial angiogenesis. Nat. Med. 1:1085-1089.

4. Baumgartner, I., et al. 1998. Constitutive expression of phVEGF165 after intramuscular gene transfer promotes collateral vessel development in patients with critical limb ischemia. Circulation. 97:1114-1123.

5. Losordo, D.W., et al. 1998. Gene therapy for myocardial angiogenesis: initial clinical results with direct myocardial injection of phVEGF165 as sole therapy for myocardial ischemia. Circulation. 98:2800-2804.

6. Folkman, J. 1995. Angiogenesis in cancer, vascular, rheumatoid and other disease. Nat. Med. 1:27-31.

7. Schaper, W., Brahander, M.D., and Lewi, P. 1971. DNA synthesis and mitoses in coronary collateral vessels of the dog. Circ. Res. 28:671-679.

8. Risau, W., et al. 1988. Vasculogenesis and angiogenesis in embryonic stem cell-derived embryoid bodies. Development. 102:471-478.

9. Risau, W. 1995. Differentiation of endothelium. FASEB J. 9:926-933.

10. Pardanaud, L., Altman, C., Kitos, P., and Dieterien-Lievre, F. 1989. Relationship between vasculogenesis, angiogenesis and haemopoiesis during avian ontogeny. Development. 105:473-485.

11. Carmeliet, P., and Collen, D. 1998. Vascular development and disorders:
} 
molecular analysis and pathogenic insights. Kidney Int. 53:1519-1549.

12. Flamme, I., and Risau, W. 1992. Induction of vasculogenesis and hematopoiesis in vitro. Development. 116:435-439.

13. Weiss, M., and Orkin, S.H. 1996. In vitro differentiation of murine embryonic stem cells: new approaches to old problems. J. Clin. Invest. 97:591-595.

14. Hatzopoulos, A.K., Folkman, J., Vasile, E., Eiselen, G.K., and Rosenberg, R.D. 1998. Isolation and characterization of endothelial progenitor cells from mouse embryos. Development. 125:1457-1468.

15. Asahara, T., et al. 1997. Isolation of putative progenitor endothelial cells for angiogenesis. Science. 275:965-967.

16. Isner, J.M., and Asahara, T. 1999. Angiogenesis and vasculogenesis as therapeutic strategies for postnatal neovascularization. J. Clin. Invest. 103:1231-1236.

17. Nakahata, T., and Ogawa, M. 1982. Hemopoietic colony-forming cells in umbilical cord blood with extensive capability to generate mono- and multipotential hemopoietic progenitors. J. Clin. Invest. 70:1324-1328.

18. Mayani, H., and Lansdorp, P.M. 1994. Thy-1 expression is linked to functional properties of primitive hematopoietic progenitor cells from human umbilical cord blood. Blood. 83:2410-2417.

19. Vaziri, H., et al. 1994. Evidence for a mitotic clock in human hematopoietic stem cells: loss of telomeric DNA with age. Proc. Natl. Acad. Sci. USA. 91:9857-9860.

20. de La Selle, V., Gluckman, E., and Bruley-Rosset, M. 1996. Newborn blood can engraft adult mice without inducing graft-versus-host disease across non $\mathrm{H}-2$ antigens. Blood. 87:3977-3983.

21. Gluckman, E., et al. 1989. Hematopoietic reconstitution in a patient with Fanconi's anemia by means of umbilical-cord blood from an HLAidentical sibling. N. Engl. J. Med. 321:1174-1178.

22. Broxmeyer, H.E., et al. 1989. Human umbilical cord blood as a potential source of transplantable hematopoietic stem/progenitor cells. Proc. Natl. Acad. Sci. USA. 86:3828-3832

23. Wagner, J.E., Kernan, N.A., Steinbuch, M., Broxmeyer, H.E., and Gluckman, E. 1995. Allogeneic sibling umbilical-cord-blood transplantation in children with malignant and non-malignant disease. Lancet. 346:214-219.

24. Kurtzberg, J., et al. 1996. Placental blood as a source of hematopoietic stem cells for transplantation into unrelated recipients. N. Engl. J. Med. 335:157-166.

25. Gluckman, E., et al. 1997. Outcome of cord-blood transplantation from related and unrelated donors. Eurocord Transplant Group and the European Blood and Marrow Transplantation Group. N. Engl. J. Med. 337:373-381.

26. Chen, C.H., et al. 1994. Automated enumeration of CD34+ cells in peripheral blood and bone marrow. J. Hematother. 3:3-13.

27. Murohara, T., Delyani, J.A., Albelda, S.M., and Lefer, A.M. 1996. Blockade of platelet endothelial cell adhesion molecule- 1 protects against myocardial ischemia and reperfusion injury in cats. J. Immunol. 156:3550-3557

28. Garlanda, C., and Dejana, E. 1997. Heterogeneity of endothelial cells. Specific markers. Arterioscler. Thromb. Vasc. Biol. 17:1193-1202.

29. Voyta, J.C., Via, D.P., Butterfield, C.E., and Zetter, B.R. 1984. Identification and isolation of endothelial cells based on their increased uptake of acetylated-low density lipoprotein. J. Cell Biol. 99:2034-2040.

30. Kojima, H., et al. 1998. Detection and imaging of nitric oxide with novel fluorescent indicators: diaminofluoresceins. Anal. Chem. 70:2446-2453.

31. Ziada, A.M., Hudlicka, O., Tyler, K.R., and Wright, A.J. 1984. The effect of long-term vasodilation on capillary growth and performance in rabbit heart and skeletal muscle. Cardiovasc. Res. 18:724-732.

32. Murohara, T., et al. 1998. Nitric oxide synthase modulates angiogenesis in response to tissue ischemia. J. Clin. Invest. 101:2567-2578.

33. Murohara, T., et al. 1998. Vascular endothelial growth factor/vascular permeability factor enhances vascular permeability via nitric oxide and prostacyclin. Circulation. 97:99-107.

34. Kroll, J., and Waltenberger, J. 1998. VEGF-A induces expression of eNOS and iNOS in endothelial cells via VEGF receptor-2 (KDR). Biochem. Biophys. Res. Commun. 252:743-746.

35. Ku, D.D., Zaleski, J.K., Liu, S., and Brock, T.A. 1993. Vascular endothelial growth factor induces EDRF-dependent relaxation in coronary arteries. Am. J. Physiol. 265:H586-H592.

36. van der Zee, R., et al. 1997. Vascular endothelial growth factor (VEGF)/vascular permeability factor (VPF) augments nitric oxide release from quiescent rabbit and human vascular endothelium. Circulation. 95:1030-1037.

37. Fulton, D., et al. 1999. Regulation of endothelium-derived nitric oxide production by the protein kinase Akt. Nature. 399:597-601.

38. Dimmeler, S., et al. 1999. Activation of nitric oxide synthase in endothelial cells by Akt-dependent phosphorylation. Nature. 399:601-605.

39. Takahashi, T., et al. 1999. Ischemia- and cytokine-induced mobilization of bone marrow-derived endothelial progenitor cells for neovascularization. Nat. Med. 5:434-438.

40. Asahara, T., et al. 1999. VEGF contributes to postnatal neovasculariza- tion by mobilizing bone marrow-derived endothelial progenitor cells. EMBO J. 18:3964-3972.

41. Nishikawa, S., et al. 1998. Progressive lineage analysis by cell sorting and culture identifies FLK1+VE-cadherin + cells at a diverging point of endothelial and hemopoietic lineages. Development. 125:1747-1757.

42. Ito, A., Nomura, S., Hirota, S., Suda, T., and Kitamura, Y. 1995. Enhanced expression of CD34 messenger RNA by developing endothelial cells of mice. Lab. Invest. 72:532-538.

43. Krause, D.S., Fackler, M.J., Civin, C.I., and May, W.S. 1996. CD34: structure, biology, and clinical utility. Blood. 87:1-13.

44. Millauer, B., et al. 1993. High affinity VEGF binding and developmental expression suggest Flk-1 as a major regulator of vasculogenesis and angiogenesis. Cell. 72:835-846.

45. Yamaguchi, T.P., Dumont, D.J., Conlon, R.A., Breitman, M.L., and Rossant, J. 1993. flk-1, and flt-related receptor tyrosine kinase is an early marker for endothelial cell precursors. Development. 118:489-498.

46. Eichmann, A., et al. 1997. Ligand-dependent development of the endothelial and hemopoietic lineages from embryonic mesodermal cells expressing vascular endothelial growth factor receptor 2. Proc. Natl. Acad. Sci. USA. 94:5141-5146.

47. Sato, T.N., et al. 1995. Distinct roles of the receptor tyrosine kinases Tie1 and Tie-2 in blood vessel formation. Nature. 376:70-74.

48. Schnurch, H., and Risau, W. 1993. Expression of tie-2, a member of a novel family of receptor tyrosine kinases, in the endothelial cell lineage. Development. 119:957-968.

49. Fina, L., et al. 1990. Expression of the CD34 gene in vascular endothelial cells. Blood. 75:2417-2426.

50. Soligo, D., et al. 1991. Identification of CD34+ cells in normal and pathological bone marrow biopsies by OBEND10 monoclonal antibody. Leukemia. 5:1026-1030.

51. Matthews, W., et al. 1991. A receptor tyrosine kinase cDNA isolated from a population of enriched primitive hematopoietic cells and exhibiting close genetic linkage to c-kit. Proc. Natl. Acad. Sci. USA. 88:9026-9030.

52. Katoh, O., Tauchi, H., Kawaishi, K., Kimura, A., and Satou, Y. 1995. Expression of the vascular endothelial growth factor (VEGF) receptor gene, KDR, in hematopoietic cells and inhibitory effect of VEGF on apoptotic cell death caused by ionizing radiation. Cancer Res. 55:5687-5692.

53. Yano, M., et al. 1997. Expression and function of murine receptor tyrosine kinases, TIE and TEK, in hematopoietic stem cells. Blood. 89:4317-4326.

54. Civin, C.I., et al. 1984. Antigenic analysis of hematopoiesis. III. A hematopoietic progenitor cell surface antigen defined by a monoclonal antibody raised against KG-1a. J. Immunol. 133:157-165.

55. Katz, F., Tindle, R.W., Sutherland, D.R., and Greaves, M.D. 1985. Identification of a membrane glycoprotein associated with hemopoietic progenitor cells. Leuk. Res. 9:191-198.

56. Andrews, R.G., Singer, J.W., and Bernstein, I.D. 1986. Monoclonal antibody $12-8$ recognizes a $115-\mathrm{kd}$ molecule present on both unipotent and multipotent hematopoietic colony-forming cells and their precursors. Blood. 67:842-845

57. Rafii, S., et al. 1995. Characterization of hematopoietic cells arising on the textured surface of left ventricular assist devices. Ann. Thorac. Surg. 60:1627-1632.

58. Shi, Q., et al. 1998. Evidence for circulating bone marrow-derived endothelial cells. Blood. 92:362-367.

59. Bhatia, M., Bonnet, D., Murdoch, B., Gan, O.I., and Dick, J.E. 1998. A newly discovered class of human hematopoietic cells with SCID-repopulating activity. Nat. Med. 4:1038-1045.

60. Rafii, S. 2000. Circulating endothelial precursors: mystery, reality, and promise. J. Clin. Invest. 105:17-19.

61. Peichev, M., et al. 2000. Expression of VEGFR-2 and AC133 by circulating human CD34(+) cells identifies a population of functional endothelial precursors. Blood. 95:952-958.

62. Solovey, A., et al. 1997. Circulating activated endothelial cells in sickle cell anemia. N. Engl. J. Med. 337:1584-1590.

63. Mutin, M., et al. 1999. Direct evidence of endothelial injury in acute myocardial infarction and unstable angina by demonstration of circulating endothelial cells. Blood. 93:2951-2958.

64. Lin, Y., Weisdorf, D.J., Solovey, A., and Hebbel, R.P. 2000. Origins of circulating endothelial cells and endothelial outgrowth from blood. J. Clin. Invest. 105:71-77.

65. Wood, H.B., May, G., Healy, L., Enver, T., and Morriss-Kay, G.M. 1997. CD34 expression patterns during early mouse development are related to modes of blood vessel formation and reveal additional sites of hematopoiesis. Blood. 90:2300-2311.

66. Dieterlen-Lievre, F., and Martin, C. 1981. Diffuse intraembryonic hemopoiesis in normal and chimeric avian development. Dev. Biol. 88:180-191.

67. Kalka, C., et al. 2000. Transplantation of ex vivo expanded endothelial progenitor cells for therapeutic neovascularization. Proc. Natl. Acad. Sci. USA. 97:3422-3427.

68. Crisa, L., et al. 1999. Human cord blood progenitors sustain thymic Tcell development and a novel form of angiogenesis. Blood. 94:3928-3940. 